\title{
Exponential Runge-Kutta methods for parabolic problems
}

\author{
Marlis Hochbruck ${ }^{\mathrm{a}, *}$, Alexander Ostermann ${ }^{\mathrm{b}}$ \\ ${ }^{a}$ Mathematisches Institut, Heinrich-Heine Universität Düsseldorf, \\ Universitätsstraße 1, D-40225 Düsseldorf, Germany \\ b Institut für Technische Mathematik, Geometrie und Bauinformatik, Universität \\ Innsbruck, Technikerstraße 13, A-6020 Innsbruck, Austria
}

\begin{abstract}
The aim of this paper is to construct exponential Runge-Kutta methods of collocation type and to analyze their convergence properties for linear and semilinear parabolic problems. For the analysis, an abstract Banach space framework of sectorial operators and locally Lipschitz continuous nonlinearities is chosen. This framework includes interesting examples like reaction-diffusion equations. It is shown that the methods converge at least with their stage order, and that convergence of higher order (up to the classical order) occurs, if the problem has sufficient temporal and spatial smoothness. The latter, however, might require the source function to fulfil unnatural boundary conditions. Therefore, the classical order is not always obtained and an order reduction must be expected, in general.
\end{abstract}

Key words: Semilinear parabolic problems; Exponential quadrature rules;

Exponential collocation methods; Convergence bounds; Preservation of equilibria

\section{Introduction}

In this paper we consider the time discretization of semilinear parabolic problems

$$
u^{\prime}(t)+A u(t)=g(t, u), \quad u\left(t_{0}\right)=u_{0}
$$

by exponential Runge-Kutta methods. We present a construction for the numerical solution of (1) based on the variation-of-constants formula. On the

* Corresponding author.

Email addresses: marlis@am.uni-duesseldorf.de (Marlis Hochbruck), alexander.ostermann@uibk.ac.at (Alexander Ostermann). 
one hand, this approach is motivated by the well-known construction of collocation methods for ordinary differential equations. On the other hand, convergence proofs for one-step discretizations of (1) are generally based on a discrete version of the variation-of-constants formula. It therefore seems to be natural to define the numerical method already with the help of the variationof-constants formula.

The idea of exponential integrators is an old one and has been proposed independently by many authors. To our knowledge, Lawson [12] was the first who combined the exponential function with explicit Runge-Kutta methods to obtain A-stability. This idea was considerably generalized in a remarkable paper by Friedly [5]. There, a class of explicit exponential Runge-Kutta methods was introduced and, based on the nonstiff order conditions, some particular methods were constructed. Similar methods were later proposed in $[3,11,19]$ and quite recently in the context of Lie groups [2]. In our paper [10], we have analyzed the convergence properties of such explicit exponential methods for semilinear parabolic problems. A different class of exponential integrators motivated by Rosenbrock methods was proposed in [9]. Exponential multistep methods were first considered by Nørsett [17] and more recently derived in [3]. Note, however, that all above mentioned papers deal with explicit methods, whereas we focus here on implicit methods.

The outline of the paper is as follows: We start with the construction of exponential Runge-Kutta methods of collocation type in Section 2. In Section 3 we study linear parabolic problems. After stating the precise assumptions on the operator $A$, we show that an $s$-stage exponential Runge-Kutta method of collocation type converges with order $\min (s+1, p)$, where $p$ denotes the order of the underlying Runge-Kutta method. For obtaining an order of convergence higher than $s+1$, the source function must satisfy unnatural boundary conditions. The lack of this condition implies a severe order reduction for many methods like the exponential Gauss methods where $p=2 s$. The situation is thus very similar to that of other one-step methods like implicit Runge-Kutta methods [13] or linearly implicit methods [14].

In Section 4, we study the convergence properties of exponential Runge-Kutta methods for semilinear problems. We work in a Banach space framework of sectorial operators with locally Lipschitz continuous nonlinearities. The framework is sufficiently general to cover interesting examples such as reactiondiffusion equations or the incompressible Navier-Stokes equations in two and three space dimensions. As in the linear case, we can prove that an $s$-stage exponential Runge-Kutta method of collocation type converges with order $s$ at least. Again, higher and even fractional order of convergence is possible if the nonlinearity evaluated on the exact solution has sufficient spatial regularity. In particular, full order of convergence can be obtained for periodic boundary conditions. As a final result, we show that exponential Runge-Kutta methods preserve equilibria of autonomous problems. 


\section{Exponential Runge-Kutta methods of collocation type}

The main idea behind exponential integrators of collocation type is to replace the function $g$ in the variation-of-constants formula

$$
u\left(t_{n}+h\right)=\mathrm{e}^{-h A} u\left(t_{n}\right)+\int_{0}^{h} \mathrm{e}^{-(h-\tau) A} g\left(t_{n}+\tau, u\left(t_{n}+\tau\right)\right) \mathrm{d} \tau
$$

by a collocation polynomial $\widehat{g}_{n}$ which yields

$$
u\left(t_{n}+h\right) \approx \mathrm{e}^{-h A} u\left(t_{n}\right)+\int_{0}^{h} \mathrm{e}^{-(h-\tau) A} \widehat{g}_{n}(\tau) \mathrm{d} \tau
$$

For this we choose non-confluent collocation nodes $c_{1}, \ldots, c_{s}$ and we assume that we are given approximations

$$
u_{n} \approx u\left(t_{n}\right), \quad U_{n, i} \approx u\left(t_{n}+c_{i} h\right) .
$$

We then define $\widehat{g}_{n}$ by the collocation conditions $\widehat{g}_{n}\left(c_{i} h\right)=g\left(t_{n}+c_{i} h, U_{n, i}\right)=$ $G_{n, i}$ so that

$$
\widehat{g}_{n}(\tau)=\sum_{j=1}^{s} \ell_{j}(\tau) G_{n, j},
$$

where $\ell_{j}$ is the Lagrange interpolation polynomial

$$
\ell_{j}(\tau)=\prod_{m \neq j} \frac{\tau / h-c_{m}}{c_{j}-c_{m}}
$$

Replacing $u\left(t_{n}\right)$ in (3) by the given approximation $u_{n}$ and evaluating the integral yields an approximation to the exact solution at time $t_{n+1}$

$$
u_{n+1}=\mathrm{e}^{-h A} u_{n}+h \sum_{i=1}^{s} b_{i}(-h A) G_{n, i}
$$

where

$$
b_{i}(-h A)=\frac{1}{h} \int_{0}^{h} \mathrm{e}^{-(h-\tau) A} \ell_{i}(\tau) \mathrm{d} \tau .
$$

It still remains to define the approximations $U_{n, i}$. For this, we use the same approach. Substituting $h$ by $c_{i} h$ in (3), we obtain

$$
U_{n, i}=\mathrm{e}^{-c_{i} h A} u_{n}+h \sum_{j=1}^{s} a_{i j}(-h A) G_{n, j}
$$

where

$$
a_{i j}(-h A)=\frac{1}{h} \int_{0}^{c_{i} h} \mathrm{e}^{-\left(c_{i} h-\tau\right) A} \ell_{j}(\tau) \mathrm{d} \tau .
$$


Since $\ell_{j}$ is a polynomial of degree at most $s-1$, the coefficients $b_{i}(-h A)$ and $a_{i j}(-h A)$ are linear combinations of the functions

$$
\varphi_{j}(-t A)=\frac{1}{t^{j}} \int_{0}^{t} \mathrm{e}^{-(t-\tau) A} \frac{\tau^{j-1}}{(j-1) !} \mathrm{d} \tau, \quad 1 \leq j \leq s .
$$

The scheme (4) is called exponential Runge-Kutta method of collocation type henceforth.

We note for later use that

$$
\varphi_{k+1}(z)=\frac{\varphi_{k}(z)-1 / k !}{z}, \quad \varphi_{k}(0)=\frac{1}{k !} .
$$

It seems worth mentioning that this construction reduces to the construction of Runge-Kutta methods with coefficients $b_{i}=b_{i}(0)$ and $a_{i j}=a_{i j}(0)$ if we consider the limit $A \rightarrow 0$. The limiting method will be called underlying Runge-Kutta method henceforth.

\section{$3 \quad$ Linear problems}

In this section, we will derive error bounds for exponential Runge-Kutta discretizations of linear parabolic problems

$$
u^{\prime}(t)+A u(t)=f(t), \quad u(0)=u_{0}
$$

with time-invariant operator $A$. Note that for such problems, exponential Runge-Kutta methods reduce to exponential quadrature rules

$$
u_{n+1}=\mathrm{e}^{-h A} u_{n}+h \sum_{i=1}^{s} b_{i}(-h A) f\left(t_{n}+c_{i} h\right)
$$

with

$$
b_{i}(-h A)=\frac{1}{h} \int_{0}^{h} \mathrm{e}^{-(h-\tau) A} \ell_{i}(\tau) \mathrm{d} \tau
$$

Our analysis of (6) will be based on an abstract formulation of (5) as an evolution equation in a Banach space $(X,\|\cdot\|)$. Let $\mathcal{D}(A)$ denote the domain of $A$ in $X$. Our basic assumptions on the operator $A$ are that of [7].

Assumption 1 Let $A: \mathcal{D}(A) \rightarrow X$ be sectorial, i.e. $A$ is a densely defined and closed linear operator on $X$ satisfying the resolvent condition

$$
\left\|(\lambda I-A)^{-1}\right\| \leq \frac{M}{|\lambda-a|}
$$


on the sector $\{\lambda \in \mathbb{C} ; \vartheta \leq|\arg (\lambda-a)| \leq \pi, \lambda \neq a\}$ for $M \geq 1, a \in \mathbb{R}$, and $0<\vartheta<\pi / 2$.

Under this assumption, the operator $-A$ is the infinitesimal generator of an analytic semigroup $\left\{\mathrm{e}^{-t A}\right\}_{t \geq 0}$. For $\omega>-a$, the fractional powers of $\widetilde{A}=A+\omega I$ are well-defined. The following stability bounds for the semigroup are crucial in our analysis.

Lemma 1 Under Assumption 1, the following bounds hold uniformly on $0 \leq$ $t \leq T$

$$
\begin{aligned}
\left\|\mathrm{e}^{-t A}\right\|+\left\|t^{\gamma} \widetilde{A}^{\gamma} \mathrm{e}^{-t A}\right\| & \leq C, \quad \gamma \geq 0 \\
\left\|h A \sum_{j=1}^{n-1} \mathrm{e}^{-j h A}\right\| & \leq C .
\end{aligned}
$$

Proof The bound (8a) is well-known from literature. In order to show (8b), we use the representation

$$
\mathrm{e}^{-h A}=\frac{1}{2 \pi i} \int_{\Gamma} \mathrm{e}^{h \lambda}(\lambda I+A)^{-1} \mathrm{~d} \lambda
$$

where $\Gamma$ is an appropriate contour in the resolvent set of $-A$. Using the resolvent identity $A(\lambda I+A)^{-1}=I-\lambda(\lambda I+A)^{-1}$ shows

$$
-h A \sum_{j=1}^{n-1} \mathrm{e}^{-j h A}=\frac{1}{2 \pi i} \int_{\Gamma} w(h \lambda) \mathrm{e}^{h \lambda}(\lambda I+A)^{-1} \mathrm{~d} \lambda
$$

with

$$
w(z)=z \frac{\mathrm{e}^{(n-1) z}-1}{\mathrm{e}^{z}-1} .
$$

Since $|w(z)| \leq C$, uniformly on $\Gamma$, we get the desired bound.

The stability estimate (8a) enables us to define the bounded operators

$$
\varphi_{j}(-t A)=\frac{1}{t^{j}} \int_{0}^{t} \mathrm{e}^{-(t-\tau) A} \frac{\tau^{j-1}}{(j-1) !} \mathrm{d} \tau, \quad j \geq 1 .
$$

In order to analyze (6), we expand the exact solution of (5) into a Taylor series

$$
\begin{aligned}
u\left(t_{n+1}\right) & =\mathrm{e}^{-h A} u\left(t_{n}\right)+\int_{0}^{h} \mathrm{e}^{-(h-\tau) A} f\left(t_{n}+\tau\right) \mathrm{d} \tau \\
& =\mathrm{e}^{-h A} u\left(t_{n}\right)+h \sum_{k=1}^{s} \varphi_{k}(-h A) h^{k-1} f^{(k-1)}\left(t_{n}\right)+\mathcal{O}\left(h^{s+1}\right) .
\end{aligned}
$$


This has to be compared with the Taylor series of the numerical solution (6)

$$
\begin{aligned}
u_{n+1} & =\mathrm{e}^{-h A} u_{n}+h \sum_{i=1}^{s} b_{i}(-h A) f\left(t_{n}+c_{i} h\right) \\
& =\mathrm{e}^{-h A} u_{n}+h \sum_{i=1}^{s} b_{i}(-h A) \sum_{k=0}^{s-1} \frac{h^{k} c_{i}^{k}}{k !} f^{(k)}\left(t_{n}\right)+\mathcal{O}\left(h^{s+1}\right) .
\end{aligned}
$$

We are now ready to state our convergence result.

Theorem 1 For the numerical solution of (5), (7), consider an exponential Runge-Kutta method (6). If $f^{(s)} \in L^{1}(0, T ; X)$, then the following error bound holds

$$
\left\|u_{n}-u\left(t_{n}\right)\right\| \leq C \cdot h^{s} \cdot \int_{0}^{t_{n}}\left\|f^{(s)}(\tau)\right\| \mathrm{d} \tau
$$

uniformly on $0 \leq t_{n} \leq T$. The constant $C$ depends on $T$, but is independent of $n$ and $h$.

Proof Let $e_{n}=u_{n}-u\left(t_{n}\right)$ denote the difference between the numerical and the exact solution. Then

$$
e_{n+1}=\mathrm{e}^{-h A} e_{n}-\delta_{n+1}
$$

with

$$
\begin{aligned}
\delta_{n+1}=\int_{0}^{h} \mathrm{e}^{-(h-\tau) A} \int_{0}^{\tau} \frac{(\tau-\xi)^{s-1}}{(s-1) !} f^{(s)}\left(t_{n}+\xi\right) \mathrm{d} \xi \mathrm{d} \tau \\
\quad-h \sum_{i=1}^{s} b_{i}(-h A) \int_{0}^{c_{i} h} \frac{\left(c_{i} h-\tau\right)^{s-1}}{(s-1) !} f^{(s)}\left(t_{n}+\tau\right) \mathrm{d} \tau .
\end{aligned}
$$

The desired bound now follows immediately from

$$
\left\|e_{n}\right\| \leq \sum_{j=0}^{n-1}\left\|\mathrm{e}^{-j h A}\right\| \cdot\left\|\delta_{n-j}\right\|
$$

and the stability bound (8a).

Remark The above theorem has a straightforward extension to variable step sizes $h_{n}=t_{n}-t_{n-1}$, where

$$
\left\|u_{n}-u\left(t_{n}\right)\right\| \leq C \sum_{j=0}^{n-1} h_{j+1}^{s} \int_{t_{j}}^{t_{j+1}}\left\|f^{(s)}(\tau)\right\| \mathrm{d} \tau .
$$

Theorem 1 is not yet optimal for methods whose underlying quadrature rule satisfies additional order conditions. The first of these conditions is that the 
underlying quadrature rule is of order $s+1$, i.e.,

$$
\sum_{i=1}^{s} b_{i}(0) c_{i}^{s}=\frac{1}{s+1}
$$

This is satisfied, for instance, by the Radau methods with $s \geq 2$ and by all Gauss methods.

Theorem 2 Under the hypotheses of Theorem 1, assume that the exponential Runge-Kutta method (6) satisfies in addition (12). If $f^{(s+1)} \in L^{1}(0, T ; X)$, then the refined error bound

$$
\left\|u_{n}-u\left(t_{n}\right)\right\| \leq C \cdot h^{s+1}\left(\left\|f^{(s)}(0)\right\|+\int_{0}^{t_{n}}\left\|f^{(s+1)}(\tau)\right\| \mathrm{d} \tau\right)
$$

holds uniformly on $0 \leq t_{n} \leq T$. The constant $C$ depends on $T$, but is independent of $n$ and $h$.

Proof The proof is very similar to that of Theorem 1. We write the defects as

$$
\delta_{n+1}=h^{s+1} \psi_{s+1}(-h A) f^{(s)}\left(t_{n}\right)+\widetilde{\delta}_{n+1}
$$

with

$$
\psi_{s+1}(-h A)=\varphi_{s+1}(-h A)-\frac{1}{s !} \sum_{i=1}^{s} b_{i}(-h A) c_{i}^{s}
$$

to get

$$
\sum_{j=0}^{n-1}\left\|\mathrm{e}^{-j h A}\right\| \cdot\left\|\widetilde{\delta}_{n-j}\right\| \leq C \cdot h^{s+1} \cdot \int_{0}^{t_{n}}\left\|f^{(s+1)}(\tau)\right\| \mathrm{d} \tau .
$$

Since the rational function $\psi_{s+1}(-h A)$ is uniformly bounded and satisfies $\psi_{s+1}(0)=0$ due to $(12)$, we infer the desired bound for

$$
\sum_{j=0}^{n-1} \mathrm{e}^{-j h A} \psi_{s+1}(-h A) f^{(s)}\left(t_{n-j-1}\right)
$$

from Lemma 2 below setting $w_{j}=\mathrm{e}^{-j h A} \psi_{s+1}(-h A)$ and $v_{j}=f^{(s)}\left(t_{j-1}\right)$. This concludes the proof.

Lemma 2 For $W_{k}=\sum_{j=0}^{k} w_{j}$ the following summation-by-parts formula holds

$$
\sum_{j=0}^{n-1} w_{j} v_{n-j}=W_{n-1} v_{1}-\sum_{j=0}^{n-2} W_{j}\left(v_{n-j-1}-v_{n-j}\right) .
$$

If the right-hand side of (5) has a higher spatial regularity, the temporal order 
of convergence can still be improved for methods satisfying

$$
\begin{gathered}
\sum_{i=1}^{s} b_{i}(0) c_{i}^{s+1}=\frac{1}{s+2} \\
\sum_{i=1}^{s} b_{i}^{\prime}(0) c_{i}^{s}=\frac{1}{(s+1)(s+2)}
\end{gathered}
$$

Lemma 3 The conditions (12) and (15) are satisfied, if the underlying quadrature rule has order $s+2$.

Proof Conditions (12) and (15a) are classical order conditions. In order to show (15b), we infer from (6b) that

$$
b_{i}^{\prime}(0)=\int_{0}^{1} \ell_{i}(\tau)(1-\tau) \mathrm{d} \tau
$$

and hence

$$
\sum_{i=1}^{s} b_{i}^{\prime}(0) c_{i}^{s}=\int_{0}^{1} \sum_{i=1}^{s} \ell_{i}(\tau) c_{i}^{s}(1-\tau) \mathrm{d} \tau=\sum_{k=1}^{s} b_{k}(0) \sum_{i=1}^{s} \ell_{i}\left(c_{k}\right) c_{i}^{s}\left(1-c_{k}\right)
$$

where the last identity follows from the fact that the quadrature rule is of order $s+1$ at least. Using $\ell_{i}\left(c_{k}\right)=\delta_{i k}$ and conditions (12) and (15a) completes the proof.

Lemma 3 shows that conditions (12) and (15) are satisfied by the Gauss methods with $s \geq 2$ and by the Radau methods with $s \geq 3$.

Theorem 3 Under the hypotheses of Theorem 2, assume that the exponential Runge-Kutta method (6) satisfies in addition (15). Further assume that $f^{(s+2)} \in L^{1}(0, T ; X)$ and let $\beta \in[0,1]$ be such that $\widetilde{A}^{\beta} f^{(s+1)} \in L^{1}(0, T ; X)$. Then the refined error bound

$$
\begin{array}{r}
\left\|u_{n}-u\left(t_{n}\right)\right\| \leq C \cdot h^{s+1+\beta}\left(\left\|\widetilde{A}^{\beta} f^{(s)}(0)\right\|+\int_{0}^{t_{n}}\left\|\widetilde{A}^{\beta} f^{(s+1)}(\tau)\right\| \mathrm{d} \tau\right) \\
+C \cdot h^{s+2}\left(\left\|f^{(s+1)}(0)\right\|+\int_{0}^{t_{n}}\left\|f^{(s+2)}(\tau)\right\| \mathrm{d} \tau\right)
\end{array}
$$

holds uniformly on $0 \leq t_{n} \leq T$. The constant $C$ depends on $T$, but is independent of $n$ and $h$.

Proof The proof is very similar to the previous two proofs. We write the defects as

$$
\delta_{n+1}=h^{s+1} \psi_{s+1}(-h A) f^{(s)}\left(t_{n}\right)+h^{s+2} \psi_{s+2}(-h A) f^{(s+1)}\left(t_{n}\right)+\widehat{\delta}_{n+1}
$$

with

$$
\psi_{s+2}(-h A)=\varphi_{s+2}(-h A)-\frac{1}{(s+1) !} \sum_{i=1}^{s} b_{i}(-h A) c_{i}^{s+1}
$$


The rational function $\psi_{s+2}(-h A)$ is uniformly bounded and satisfies $\psi_{s+2}(0)=$ 0 due to (15a). The second and third term of (16) are thus bounded as in the previous theorem. For the term with $\psi_{s+1}$, we use the fact that due to (12) and $(15 b)$

$$
\psi_{s+1}(-h A)=\psi_{s+1}(-h A)-\psi_{s+1}(0)=h A \cdot \psi_{s+1}^{(1)}(-h A)
$$

with $\psi_{s+1}^{(1)}(0)=0$. Thus, we have

$$
h^{s+1} \psi_{s+1}(-h A) f^{(s)}\left(t_{n}\right)=h^{s+1+\beta} \psi_{s+1}^{(1)}(-h A)(h \widetilde{A})^{1-\beta} \cdot A \widetilde{A}^{-1} \cdot \widetilde{A}^{\beta} f^{(s)}\left(t_{n}\right) .
$$

With the help of Lemma 2, this term can be bounded in the desired way, which concludes the proof.

Remark The restriction to $\beta \leq 1$ in Theorem 3 was made just for simplicity. If the source term has higher spatial regularity, and if further conditions of the type (15) are fulfilled, then we can also show higher temporal order of convergence. The additional conditions can be derived by expanding the defect in (16) even further, and it can be shown as in Lemma 3 that they are implied by the underlying quadrature rule being of higher order. In particular, full (classical) order is achieved for sufficiently smooth source term with periodic boundary conditions.

Example To illustrate the sharpness of the bounds in Theorem 3, we consider the linear parabolic problem

$$
\frac{\partial U}{\partial t}(x, t)-\frac{\partial^{2} U}{\partial x^{2}}(x, t)=(2+x(1-x)) \mathrm{e}^{t}
$$

for $x \in[0,1]$ and $t \in[0,1]$, subject to homogeneous Dirichlet boundary conditions. For the initial value $x(1-x)$, the exact solution is $U(x, t)=x(1-x) \mathrm{e}^{t}$.

We discretize this problem in space by standard finite differences, and in time by the exponential 2-stage Gauss method, respectively. The numerically observed temporal orders of convergence in different norms are displayed in Table 1.

Table 1

\begin{tabular}{r||c|c|c|c}
\multicolumn{1}{c||}{$N$} & $H^{1}$ & $L^{1}$ & $L^{2}$ & $L^{\infty}$ \\
\hline 50 & 2.80 & 3.53 & 3.27 & 3.00 \\
\hline 100 & 2.76 & 3.50 & 3.26 & 3.01 \\
\hline 200 & 2.75 & 3.50 & 3.25 & 3.00
\end{tabular}

Numerically observed temporal orders of convergence in different norms for discretizations with $N$ spatial degrees of freedom and $h=1 / 128$.

The attainable value of $\beta$ in Theorem 3 relies on the characterization of the domains of fractional powers of elliptic operators. The source function in (17) 
is spatially smooth but does not satisfy the boundary conditions. For the choice $X=L^{2}$, the best value is $\beta=1 / 4-\varepsilon$ for arbitrary $\varepsilon>0$, see [6]. This explains the observed orders in the discrete $L^{2}$-norm in Table 1 . The results in the other norms can be explained in a similar way, see [13, discussion after Theorem 3.3].

\section{Semilinear problems}

In this section, we study the convergence properties of exponential RungeKutta methods for semilinear parabolic problems (1). We work again in an abstract Banach space framework and assume that the linear operator $A$ satisfies Assumption 1. Our basic assumptions on $g$ are that of [7] and [18]. We thus choose $0 \leq \alpha<1$ and define $V=\mathcal{D}\left(\widetilde{A}^{\alpha}\right)$ where $\widetilde{A}$ denotes the shifted operator $\widetilde{A}=A+\omega I$. The linear space $V$ is a Banach space with norm $\|v\|_{V}=\left\|\widetilde{A}^{\alpha} v\right\|$. Note that this definition does not depend on $\omega$, since different choices of $\omega$ lead to equivalent norms. Our main hypothesis on the nonlinearity $g$ is the following.

Assumption 2 Let $g:[0, T] \times V \rightarrow X$ be locally Lipschitz-continuous. Thus there exists a real number $L(R, T)$ such that

$$
\|g(t, v)-g(t, w)\| \leq L\|v-w\|_{V}
$$

for all $t \in[0, T]$ and $\max \left(\|v\|_{V},\|w\|_{V}\right) \leq R$

We note that for the convergence proofs below, it is sufficient that (18) holds in a strip along the exact solution.

Example It is well known that reaction-diffusion equations fit in this abstract framework, as well as the incompressible Navier-Stokes equations in two and three space dimensions, see e.g., [7, Chapter 3] and [16, Section 7.3].

In order to simplify the notation, we set $f(t)=g(t, u(t))$. Inserting the exact solution into the numerical scheme gives

$$
\begin{aligned}
u\left(t_{n}+c_{i} h\right) & =\mathrm{e}^{-c_{i} h A} u\left(t_{n}\right)+h \sum_{j=1}^{s} a_{i j}(-h A) f\left(t_{n}+c_{j} h\right)+\Delta_{n, i}, \\
u\left(t_{n+1}\right) & =\mathrm{e}^{-h A} u\left(t_{n}\right)+h \sum_{i=1}^{s} b_{i}(-h A) f\left(t_{n}+c_{i} h\right)+\delta_{n+1}
\end{aligned}
$$


with the defects

$$
\begin{aligned}
\Delta_{n, i}= & \int_{0}^{c_{i} h} \mathrm{e}^{-\left(c_{i} h-\tau\right) A} \int_{0}^{\tau} \frac{(\tau-\xi)^{s-1}}{(s-1) !} f^{(s)}\left(t_{n}+\xi\right) \mathrm{d} \xi \mathrm{d} \tau \\
& -h \sum_{j=1}^{s} a_{i j}(-h A) \int_{0}^{c_{j} h} \frac{\left(c_{j} h-\tau\right)^{s-1}}{(s-1) !} f^{(s)}\left(t_{n}+\tau\right) \mathrm{d} \tau \\
\delta_{n+1}= & \int_{0}^{h} \mathrm{e}^{-(h-\tau) A} \int_{0}^{\tau} \frac{(\tau-\xi)^{s-1}}{(s-1) !} f^{(s)}\left(t_{n}+\xi\right) \mathrm{d} \xi \mathrm{d} \tau \\
& -h \sum_{i=1}^{s} b_{i}(-h A) \int_{0}^{c_{i} h} \frac{\left(c_{i} h-\tau\right)^{s-1}}{(s-1) !} f^{(s)}\left(t_{n}+\tau\right) \mathrm{d} \tau .
\end{aligned}
$$

We note for later use that

$$
h^{\alpha}\left\|\Delta_{n, i}\right\|_{V}+\left\|\widetilde{A}^{-\alpha} \delta_{n+1}\right\|_{V} \leq C \cdot h^{s} \int_{t_{n}}^{t_{n}+h}\left\|f^{(s)}(\tau)\right\|_{X} \mathrm{~d} \tau .
$$

For our convergence results below, we will always assume that (1) has a sufficiently smooth solution $u:[0, T] \rightarrow V$ and that the composition

$$
f:[0, T] \rightarrow X: t \mapsto f(t)=g(t, u(t))
$$

is a smooth mapping, too. The latter is the case if $g:[0, T] \times V \rightarrow X$ is sufficiently often Fréchet differentiable. Our error bounds will be given in terms of derivatives of $f$.

Theorem 4 Let the initial value problem (1) satisfy Assumptions 1 and 2 and consider for its solution an exponential Runge-Kutta method of collocationtype (4). Then, for h sufficiently small, there exists a unique numerical solution $u_{n}(0 \leq n h \leq T)$ whose error satisfies

$$
\left\|u_{n}-u\left(t_{n}\right)\right\|_{V} \leq C \cdot h^{s} \sup _{0 \leq \tau \leq T}\left\|f^{(s)}(\tau)\right\|_{X}
$$

provided that the right-hand side is bounded. The constant $C$ depends on $T$, but is independent of $n$ and $h$.

Proof Let $e_{n}=u_{n}-u\left(t_{n}\right)$ and $E_{n, i}=U_{n, i}-u\left(t_{n}+c_{i} h\right)$ denote the difference between the numerical and the exact solution. Subtracting (19) from the numerical scheme gives the error recursion

$$
\begin{aligned}
& E_{n, i}=\mathrm{e}^{-c_{i} h A} e_{n}+h \sum_{j=1}^{s} a_{i j}(-h A)\left(g\left(t_{n}+c_{j} h, U_{n, j}\right)-f\left(t_{n}+c_{j} h\right)\right)-\Delta_{n, i}, \\
& e_{n+1}=\mathrm{e}^{-h A} e_{n}+h \sum_{i=1}^{s} b_{i}(-h A)\left(g\left(t_{n}+c_{i} h, U_{n, i}\right)-f\left(t_{n}+c_{i} h\right)\right)-\delta_{n+1} .
\end{aligned}
$$


The errors of the internal stages fulfil the preliminary estimate

$$
\left\|E_{n, i}\right\|_{V} \leq C\left\|e_{n}\right\|_{V}+C L \cdot h^{1-\alpha} \sum_{j=1}^{s}\left\|E_{n, j}\right\|_{V}+\left\|\Delta_{n, i}\right\|_{V}
$$

and thus for $h$ sufficiently small

$$
\left\|E_{n, i}\right\|_{V} \leq C\left(\left\|e_{n}\right\|_{V}+\sum_{j=1}^{s}\left\|\Delta_{n, j}\right\|_{V}\right)
$$

Solving the error recursion (23) and using (8a) gives

$$
\begin{gathered}
\left\|e_{n}\right\|_{V} \leq C \cdot h \sum_{j=1}^{n-1} t_{j}^{-\alpha} \sum_{i=1}^{s}\left\|E_{n-1-j, i}\right\|_{V}+C \cdot h^{1-\alpha} \sum_{i=1}^{s}\left\|E_{n-1, i}\right\|_{V} \\
+C \cdot h \sum_{j=1}^{n-1} t_{j}^{-\alpha}\left\|\widetilde{A}^{-\alpha} \delta_{n-j} / h\right\|_{V}+C \cdot\left\|\delta_{n}\right\|_{V} .
\end{gathered}
$$

Inserting then (24) and (21) into (25) and applying the discrete Gronwall Lemma 4 with $\rho=\alpha$ and $\sigma=0$ finally shows the desired result.

It still remains to show that the unique numerical solution exists for $h$ sufficiently small. This is shown by a standard induction argument. One considers fixed-point iteration for (4c) as long as the numerical solution stays sufficiently close to the exact solution. This allows to control the Lipschitz constant $L$ of $g$. The contraction property then turns out to be valid as long as $L h^{1-\alpha}$ is sufficiently small. We omit the details.

Lemma 4 For $h>0$ and $T>0$, let $0 \leq t_{n}=n h \leq T$. Further assume that the sequence of non-negative numbers $\varepsilon_{n}$ satisfies the inequality

$$
\varepsilon_{n} \leq a h \sum_{\nu=1}^{n-1} t_{n-\nu}^{-\rho} \varepsilon_{\nu}+b t_{n}^{-\sigma}
$$

for $0 \leq \rho<1$ and $a, b \geq 0$. Then the following estimate holds

$$
\varepsilon_{n} \leq C b t_{n}^{-\sigma} \quad \text { for } \quad 0 \leq \sigma<1
$$

where the constant $C$ depends on $\rho, \sigma, a$, and on $T$.

Proof This can be shown by using similar arguments as in the proof of Theorem 1.5.5 in [1]. We omit the details.

If the underlying Runge-Kutta method has order $s+1$, we obtain the following improved convergence result.

Theorem 5 Under the hypotheses of Theorem 4, assume that the exponential Runge-Kutta method (4) satisfies in addition (12). If $f:[0, T] \rightarrow V$ is $s+1$ 
times differentiable with $f^{(s+1)} \in L^{1}(0, T ; V)$, then the refined error bound

$$
\left\|u_{n}-u\left(t_{n}\right)\right\|_{V} \leq C \cdot h^{s+1}\left(\left\|f^{(s)}(0)\right\|_{V}+\int_{0}^{t_{n}}\left\|f^{(s+1)}(\tau)\right\|_{V} \mathrm{~d} \tau\right)
$$

holds uniformly on $0 \leq t_{n} \leq T$. The constant $C$ depends on $T$, but is independent of $n$ and $h$.

Proof The proof is very similar to the previous one. Again one has to solve the error recursion

$$
e_{n+1}=\mathrm{e}^{-h A} e_{n}+h \sum_{i=1}^{s} b_{i}(-h A)\left(g\left(t_{n}+c_{i} h, U_{n, i}\right)-f\left(t_{n}+c_{i} h\right)\right)-\delta_{n+1} .
$$

Due to our assumptions, the defects of the internal stages now satisfy the bound

$$
\left\|\Delta_{n, i}\right\|_{V} \leq C \cdot h^{s+1} \sup _{0 \leq \tau \leq h}\left\|f^{(s)}\left(t_{n}+\tau\right)\right\|_{V}
$$

The term with $\delta_{n+1}$ is estimated with the help of Lemma 2 as in the proof of Theorem 2.

Example To illustrate the bounds in Theorem 5, we consider the semilinear parabolic problem

$$
\frac{\partial U}{\partial t}(x, t)-\frac{\partial^{2} U}{\partial x^{2}}(x, t)=\frac{1}{1+U(x, t)^{2}}+\phi(x, t)
$$

for $x \in[0,1]$ and $t \in[0,1]$, subject to homogeneous Dirichlet boundary conditions. The source function $\phi$ is chosen in such a way that the exact solution of the problem is $U(x, t)=x(1-x) \mathrm{e}^{t}$.

We discretize this problem in space by standard finite differences with 200 grid points, and in time by various methods with step size $h=1 / 32$, respectively.

\begin{tabular}{|c|c|c|c|}
\hline $\begin{array}{c}\text { Gauß } \\
s=1\end{array}$ & $\begin{array}{l}\text { Gauß } \\
s=2\end{array}$ & $\begin{array}{c}\text { Radau } \\
s=2\end{array}$ & $\begin{array}{c}\text { Radau } \\
s=3\end{array}$ \\
\hline 2.02 & 3.01 & 3.03 & 4.06 \\
\hline
\end{tabular}
The numerically observed temporal orders of convergence in the maximum norm are displayed in Table 2.

Table 2

Numerically observed temporal orders of convergence in the maximum norm with 200 grid points and $h=1 / 32$.

The temporal order of convergence can be improved further under slightly stronger assumptions on the spatial regularity. In [15] similar conditions have been used for analyzing Runge-Kutta discretizations of quasilinear parabolic problems. 
Assumption 3 Let $g:[0, T] \times V \rightarrow X$ be Fréchet differentiable with respect to the second variable with locally Lipschitz-continuous derivative. Thus there exists a real number $\widetilde{L}(R, T)$ such that

$$
\left\|g(t, v)-g(t, w)-\frac{\partial g}{\partial u}(t, u)(v-w)\right\|_{X} \leq \widetilde{L}\left(\|u-v\|_{V}+\|u-w\|_{V}\right)\|v-w\|_{V}
$$

for all $t \in[0, T]$ and $\max \left(\|u\|_{V},\|v\|_{V},\|w\|_{V}\right) \leq R$.

Further, let $\beta \in[0,1]$ be such that

$$
\left\|\widetilde{A}^{\beta} \frac{\partial g}{\partial u}(t, u(t)) w\right\|_{X} \leq C\left\|\widetilde{A}^{\beta} w\right\|_{V}, \quad 0 \leq t \leq T,
$$

uniformly for all $w \in \mathcal{D}\left(\widetilde{A}^{\alpha+\beta}\right)$.

Example We illustrate the meaning of the above assumptions with the following simple example

$$
\frac{\partial U}{\partial t}(x, t)-\Delta U(x, t)=\sum_{j=1}^{d} \gamma_{j} \frac{\partial U}{\partial x_{j}}(x, t), \quad \gamma_{j} \in \mathbb{R},
$$

on a smoothly bounded domain $\Omega \subset \mathbb{R}^{d}$, subject to homogeneous Dirichlet boundary conditions. Choosing $X=L^{2}(\Omega)$ and $V=H_{0}^{1}(\Omega)$, this equation can be written in the abstract form (1) with $A=-\Delta$ and $g(u)=\sum_{j=1}^{d} \gamma_{j} \partial_{x_{j}} u$. For this example, condition (27) is obvious. In order to show (28), we use the continuity of $\partial_{x_{j}}: H^{1+\sigma}(\Omega) \rightarrow H^{\sigma}(\Omega)$ for arbitrary $\sigma \in \mathbb{R}$, see, e.g., [20, Corollary 25.1]. Since $\sum_{j=1}^{d} \gamma_{j} \partial_{x_{j}} w$ in general does not satisfy boundary conditions, the best value is $\beta=1 / 4-\varepsilon$ for arbitrary $\varepsilon>0$, see [6].

For periodic boundary conditions, the above assumptions are satisfied with $\beta=1$ for spatially smooth solutions.

We are now in the position to give the refined error estimate.

Theorem 6 Under the hypotheses of Theorem 5 and Assumption 3, suppose that the underlying Runge-Kutta method has order $s+2$. Further assume that $f:[0, T] \rightarrow V$ is $s+2$ times differentiable with $f^{(s+2)} \in L^{1}(0, T ; V)$, and $\widetilde{A}^{\beta} f^{(s)} \in L^{\infty}(0, T ; V)$. Then the refined error bound

$$
\begin{array}{r}
\left\|u_{n}-u\left(t_{n}\right)\right\|_{V} \leq C \cdot h^{s+1+\beta} \cdot \sup _{0 \leq \tau \leq t_{n}}\left\|\widetilde{A}^{\beta} f^{(s)}(\tau)\right\|_{V}+C \cdot h^{s+2}\left(\left\|f^{(s)}(0)\right\|_{V}\right. \\
\left.+\int_{0}^{t_{n}}\left\|f^{(s+1)}(\tau)\right\|_{V} \mathrm{~d} \tau+\int_{0}^{t_{n}}\left\|f^{(s+2)}(\tau)\right\|_{V} \mathrm{~d} \tau\right)
\end{array}
$$

holds uniformly on $0 \leq t_{n} \leq T$. The constant $C$ depends on $T$ and on the size of $\sup _{0 \leq t \leq T}\left\|u^{\prime}(t)\right\|_{V}$, but is independent of $n$ and $h$. 
Proof The proof is very similar to the previous ones. We therefore concentrate on those aspects that go beyond the ideas used in the previous proofs.

The defect of the internal stages is now written as

$$
\Delta_{n, i}=h^{s+1} \psi_{i, s+1}(-h A) f^{(s)}\left(t_{n}\right)+\widetilde{\Delta}_{n, i}
$$

with

$$
\psi_{i, s+1}(-h A)=\varphi_{s+1}\left(-c_{i} h A\right) c_{i}^{s+1}-\frac{1}{s !} \sum_{j=1}^{s} a_{i j}(-h A) c_{j}^{s} .
$$

Since the underlying Runge-Kutta method has order $s+2$, we have

$$
\sum_{i=1}^{s} b_{i}(0) \psi_{i, s+1}(0)=0
$$

The error recursion $(23 \mathrm{~b})$ is now written as

$$
\begin{aligned}
e_{n+1}= & \mathrm{e}^{-h A} e_{n}+h \sum_{i=1}^{s} b_{i}(-h A) J_{n} E_{n, i}-\delta_{n+1} \\
& +h \sum_{i=1}^{s} b_{i}(-h A)\left(g\left(t_{n}+c_{i} h, U_{n, i}\right)-f\left(t_{n}+c_{i} h\right)-J_{n} E_{n, i}\right)
\end{aligned}
$$

with

We consider first the term

$$
J_{n}=\frac{\partial g}{\partial u}\left(t_{n}, u\left(t_{n}\right)\right)
$$

$$
\begin{aligned}
h \sum_{i=1}^{s} b_{i} & -h A) J_{n} \Delta_{n, i}=h \sum_{i=1}^{s} b_{i}(0) J_{n} h^{s+1} \psi_{i, s+1}(0) f^{(s)}\left(t_{n}\right) \\
& +h \sum_{i=1}^{s} b_{i}(-h A) J_{n} h^{s+1}\left(\psi_{i, s+1}(-h A)-\psi_{i, s+1}(0)\right) f^{(s)}\left(t_{n}\right) \\
& +h \sum_{i=1}^{s}\left(b_{i}(-h A)-b_{i}(0)\right) J_{n} h^{s+1} \psi_{i, s+1}(0) f^{(s)}\left(t_{n}\right) \\
& +h \sum_{i=1}^{s} b_{i}(-h A) J_{n} \widetilde{\Delta}_{n, i} .
\end{aligned}
$$

Due to (32), the right-hand side of (34a) vanishes. For (34b), we use

$$
\begin{aligned}
& \left(\psi_{i, s+1}(-h A)-\psi_{i, s+1}(0)\right) f^{(s)}\left(t_{n}\right) \\
& \quad=h^{\beta}\left(\psi_{i, s+1}(-h A)-\psi_{i, s+1}(0)\right)(h \widetilde{A})^{-\beta} \cdot \widetilde{A}^{\beta} f^{(s)}\left(t_{n}\right),
\end{aligned}
$$

whereas $(34 \mathrm{c})$ is rewritten as

$$
h^{s+2+\beta}(h \widetilde{A})^{-\beta} \sum_{i=1}^{s}\left(b_{i}(-h A)-b_{i}(0)\right) \widetilde{A}^{\beta} J_{n} \psi_{i, s+1}(0) f^{(s)}\left(t_{n}\right) .
$$


The convolution of these terms with $\mathrm{e}^{-j h A}$ is estimated as before and yields with the help of (28) the $h^{s+1+\beta}$-bound of (29). The $h^{s+2}$-bound for (34d) is straightforward due to the additional power of $h$ in $\widetilde{\Delta}_{n, i}$.

Next, we consider

$$
h \sum_{i=1}^{s} b_{i}(-h A) J_{n}\left(E_{n, i}+\Delta_{n, i}\right) .
$$

Inserting (23a) into (37) shows that the dominant error in this term comes from

$$
h \sum_{i=1}^{s} b_{i}(-h A) J_{n} \cdot h \sum_{m=1}^{s} a_{i m}(-h A) J_{n} \Delta_{n, m},
$$

so that it remains to bound

$$
h \sum_{i=1}^{s} b_{i}(-h A) J_{n} \cdot h \sum_{m=1}^{s} a_{i m}(-h A) J_{n} \cdot h^{s+1} \psi_{m, s+1}(-h A) f^{(s)}\left(t_{n}\right) .
$$

Since

$$
\begin{aligned}
& h a_{i m}(-h A) J_{n} h^{s+1} \psi_{m, s+1}(-h A) f^{(s)}\left(t_{n}\right) \\
& \quad=h^{s+2} a_{i m}(-h A) \cdot \widetilde{A}^{-\beta} \cdot \widetilde{A}^{\beta} J_{n} \psi_{m, s+1}(-h A) f^{(s)}\left(t_{n}\right) \\
& \quad=h^{s+1+\beta} a_{i m}(-h A)(h \widetilde{A})^{\alpha-\beta} \cdot h^{1-\alpha} \widetilde{A}^{-\alpha} \cdot \widetilde{A}^{\beta} J_{n} \psi_{m, s+1}(-h A) f^{(s)}\left(t_{n}\right),
\end{aligned}
$$

we get the desired bound for $\beta \geq \alpha$ from (38a) and for $\beta<\alpha$ from (38b), respectively.

Finally, the last term of (33) is bounded with the help of (27) and the obvious bound

$$
\left\|E_{n, i}\right\|_{V}+\int_{t_{n}}^{t_{n}+h}\left\|u^{\prime}(\tau)\right\|_{V} \leq \widetilde{C} \cdot h
$$

which follows from Theorem 5. This concludes the proof.

Remark The restriction to $\beta \leq 1$ in Theorem 6 was made just for simplicity. If the solution has higher spatial regularity, and if further conditions of the type (27) and (28) are fulfilled, then we can also show higher temporal order of convergence (up to the classical order of the underlying Runge-Kutta method). In particular, full order can be obtained for periodic boundary conditions.

Finally, we show that all exponential Runge-Kutta methods of collocation type preserve equilibrium points of the autonomous problem

$$
u^{\prime}+A u=g(u)
$$

Lemma 5 Let (39) satisfy Assumptions 1 and 2 , and let $u^{\star}$ be an equilibrium point of (39). Then, $u^{\star}$ is a fixed point of (4), i.e., if $u_{0}=u^{\star}$, then $u_{n}=u^{\star}$ for all $n \geq 1$. 
Proof Since $u(t) \equiv u^{\star}$ is a solution of (39), the variation-of-constants formula shows

$$
u^{\star}=\mathrm{e}^{-c_{i} h A} u^{\star}+\int_{0}^{c_{i} h} \mathrm{e}^{-\left(c_{i} h-\tau\right) A} g\left(u^{\star}\right) \mathrm{d} \tau=\mathrm{e}^{-c_{i} h A} u^{\star}+c_{i} h \varphi_{1}\left(-c_{i} h A\right) g\left(u^{\star}\right) .
$$

For the numerical solution we obtain from (4c)

$$
U_{n, i}=\mathrm{e}^{-c_{i} h A} u^{\star}+h \sum_{j=1}^{s} a_{i j}(-h A) g\left(U_{n, j}\right) .
$$

Since all exponential collocation methods integrate constant functions $g$ exactly, we have

$$
c_{i} h \varphi_{1}\left(-c_{i} h A\right)=h \sum_{j=1}^{s} a_{i j}(-h A) .
$$

The local uniqueness of the numerical solution therefore shows that $U_{n, i}=u^{\star}$. The result now follows from the identity

$$
\varphi_{1}(-h A)=\sum_{i=1}^{s} b_{i}(-h A)
$$

by using (4a).

\section{$5 \quad$ Concluding remarks}

In this paper we constructed and analyzed exponential Runge-Kutta methods of collocation type. The equations (4) defining the numerical scheme are implicit in the internal stages $U_{n, i}$ and moreover they require the evaluation of products of matrix functions with vectors. Therefore, we want to add some comments on the numerical implementation. In contrast to fully implicit Runge-Kutta methods, the nonlinear equations (4c) can be solved by a few steps of fixed-point iteration. This can be seen by the same arguments as those used to prove the existence of a numerical solution in the proof of Theorem 4. However, it is also possible to construct explicit schemes as in $[3,11]$. We have analyzed these methods in [10]. In particular, we showed that there exist $s$-stage explicit methods of order $s$ for methods up to $s=3$ in the framework of this paper, but that $s \geq 5$ is necessary to achieve order 4 .

For periodic boundary conditions, products of matrix functions with vectors can be computed by fast Fourier transformations. For more general boundary conditions, Krylov subspace methods, e.g. the Lanczos method, the Arnoldi method, or the Chebyshev method, can be used, see $[4,8]$.

Numerical comparisons with standard methods like multistep methods and implicit Runge-Kutta methods will be presented elsewhere. 


\section{References}

[1] H. Brunner and P.J. van der Houwen. The Numerical Solution of Volterra Equations. North-Holland, Amsterdam, 1986.

[2] E. Celledoni, A. Marthinsen, B. Owren. Commutator-free Lie group methods. FGCS, 19:341-352, 2003.

[3] S.M. Cox and P.C. Matthews. Exponential time differencing for stiff systems. Journal of Computational Physics, 176:430-455, 2002.

[4] V.L. Druskin and L.A. Knizhnerman. Krylov subspace approximations of eigenpairs and matrix functions in exact and computer arithmetic. Numer. Lin. Alg. Appl., 2:205-217, 1995.

[5] A. Friedli. Verallgemeinerte Runge-Kutta Verfahren zur Lösung steifer Differentialgleichungssysteme. In: R. Bulirsch, R.D. Grigorieff, J. Schröder (Eds.), Numerical Treatment of Differential Equations, Lecture Notes in Mathematics 631, Springer, Berlin, 1978.

[6] D. Fujiwara. Concrete characterization of the domains of fractional powers of some elliptic differential operators of the second order. Proc. Japan Acad., 43:8286, 1967.

[7] D. Henry. Geometric Theory of Semilinear Parabolic Equations. Lecture Notes in Mathematics 840, Springer, Berlin, 1981.

[8] M. Hochbruck, Ch. Lubich. On Krylov subspace approximations to the matrix exponential operator. SIAM J. Numer. Anal., 34:1911-1925, 1997.

[9] M. Hochbruck, Ch. Lubich, H. Selhofer. Exponential integrators for large systems of differential equations. SIAM J. Sci. Comput., 19:1552-1574, 1998.

[10] M. Hochbruck, A. Ostermann. Explicit exponential Runge-Kutta methods for semilinear parabolic problems. Preprint, 2004.

[11] S. Krogstad. Generalized integrating factor methods for stiff PDEs. Preprint, University of Bergen, 2003.

[12] J.D. Lawson. Generalized Runge-Kutta processes for stable systems with large Lipschitz constants. SIAM J. Numer. Anal., 4:372-380, 1967.

[13] Ch. Lubich, A. Ostermann. Runge-Kutta methods for parabolic equations and convolution quadrature. Math. Comp., 60:105-131, 1993.

[14] Ch. Lubich, A. Ostermann. Linearly implicit time discretization of non-linear parabolic equations. IMA J. Numer. Anal., 15:555-583, 1995.

[15] Ch. Lubich, A. Ostermann. Runge-Kutta approximation of quasi-linear parabolic equations. Math. Comp., 64:601-627, 1995.

[16] A. Lunardi. Analytic Semigroups and Optimal Regularity in Parabolic Problems. Birkhäuser, Basel, 1995. 
[17] S.P. Nørsett. An A-stable modification of the Adams-Bashforth methods. In: J.L. Morris (Ed.), Conference on the Numerical Solution of Differential Equations, Lecture Notes in Mathematics 109, Springer, Berlin, 1978.

[18] A. Pazy. Semigroups of Linear Operators and Applications to Partial Differential Equations. Springer, New York, 1983.

[19] K. Strehmel, R. Weiner. B-convergence results for linearly implicit one step methods. BIT, 27:264-281, 1987.

[20] F. Treves. Basic Linear Partial Differential Equations. Academic Press, New York, 1975. 